\title{
Optimal Beam Loading in a Laser-Plasma Accelerator
}

\author{
Manuel Kirchen $\odot,{ }^{1, *}$ Sören Jalas $\odot,{ }^{1}$ Philipp Messner, ${ }^{2,1}$ Paul Winkler, ${ }^{3,1}$ Timo Eichner, ${ }^{1}$ Lars Hübner, ${ }^{3,1}$ \\ Thomas Hülsenbusch, ${ }^{3,1}$ Laurids Jeppe $\odot,{ }^{1}$ Trupen Parikh, ${ }^{3}$ Matthias Schnepp, ${ }^{1}$ and Andreas R. Maier $\circledast^{3,1}$ \\ ${ }^{1}$ Center for Free-Electron Laser Science and Department of Physics Universität Hamburg, \\ Luruper Chaussee 149, 22761 Hamburg, Germany \\ ${ }^{2}$ International Max Planck Research School for Ultrafast Imaging and Structural Dynamics, \\ Luruper Chaussee 149, 22761 Hamburg, Germany \\ ${ }^{3}$ Deutsches Elektronen Synchrotron (DESY), Notkestraße 85, 22607 Hamburg, Germany
}

(Received 11 August 2020; revised 16 December 2020; accepted 2 March 2021; published 26 April 2021)

\begin{abstract}
Applications of laser-plasma accelerators demand low energy spread beams and high-efficiency operation. Achieving both requires flattening the accelerating fields by controlled beam loading of the plasma wave. Here, we optimize the generation of an electron bunch via localized ionization injection, such that the combination of injected current profile and averaged acceleration dynamics results in optimal beam loading conditions. This enables the reproducible production of $1.2 \% \mathrm{rms}$ energy spread bunches with $282 \mathrm{MeV}$ and $44 \mathrm{pC}$ at an estimated energy-transfer efficiency of $\sim 19 \%$. We correlate shot-to-shot variations to reveal the phase space dynamics and train a neural network that predicts the beam quality as a function of the drive laser.
\end{abstract}

DOI: 10.1103/PhysRevLett.126.174801

In a laser-plasma accelerator (LPA) [1,2], an intense laser pulse propagates through ionized gas and excites a trailing electron density wave, the plasma wakefield, which can trap and accelerate electrons from the plasma background to $\mathrm{GeV}$ energies over centimeter distances [3,4]. LPAs produce high-brightness electron bunches of few-femtosecond duration with kiloampere peak currents $[5,6]$ and submicron emittances $[7,8]$, which makes them promising candidates for driving a compact free-electron laser $[9,10]$ or future linear collider [11]. However, delivering the small energy spreads required by applications remains a challenge, in particular, since the steep gradients of the fieldswhich are inherent to the small size of the accelerating structure-can imprint large correlated momentum spreads onto the accelerated bunch.

At the same time, to operate LPAs efficiently [12], the accelerated electrons have to absorb a significant fraction of the energy stored in the plasma wave, which reduces the accelerating field along the bunch through an effect known as beam loading [13-16]. By shaping the bunch current profile, beam loading can be used to locally flatten the longitudinal wakefield. To effectively prevent the accumulation of correlated energy spread, however, it is important to further consider the full dynamic evolution of the plasma

Published by the American Physical Society under the terms of the Creative Commons Attribution 4.0 International license. Further distribution of this work must maintain attribution to the author(s) and the published article's title, journal citation, and DOI. wakefield and phase of the injected bunch. Carefully optimizing those dynamics can result in optimal beam loading conditions that enable both efficient acceleration and the preservation of a small initial energy spread.

Previous experiments first observed effects from beam loading in a laser-plasma accelerator $[17,18]$, studied the limit of fully loaded wakefields [19], presented evidence of loading optimum total charges [20], and investigated the transition from beam-loaded to beam-dominated wakefields [21]. Albeit producing high charge beams ( 300 pC) by operating in regimes of stronger blowout, previous work $[20,21]$ did not yet fully exploit the mechanism that leads to flat accelerating fields and, consequently, reported large energy spreads $(\sim 15 \%)$.

In this Letter, we experimentally demonstrate optimal beam loading in a laser-plasma accelerator. Electron bunches with a small initial slice energy spread are generated from localized ionization injection [22-24] and accelerated to hundreds of $\mathrm{MeV}$ in a tailored plasma source. Tuning of the beam loading - by balancing laser and plasma parameters to optimize the combination of injected bunch current profile and acceleration dynamicsleads to an on-average flat accelerating field experienced by the electrons which minimizes the projected energy spread to the $1 \%$ level.

From an analysis of shot-to-shot variations, we show that deviating from these optimal conditions increases the energy spread by imprinting a positive or negative correlation onto the longitudinal phase space. Using machine learning, we build a single-shot predictive model which links and quantifies the sensitivity of this process to 


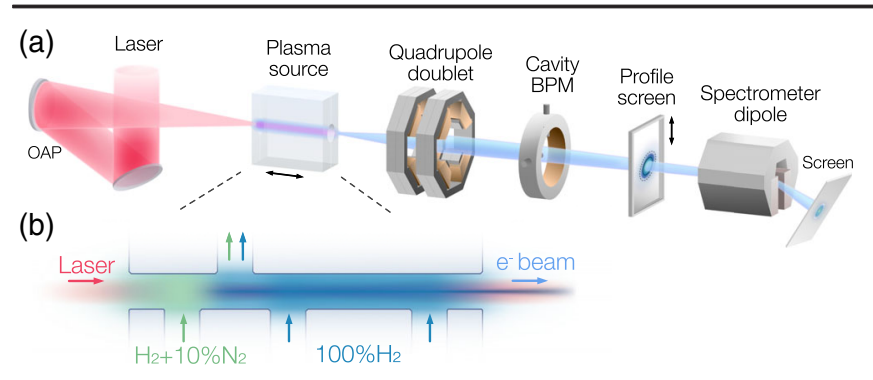

FIG. 1. Laser-plasma accelerator. (a) Schematic of the LuX laser and electron beam line. (b) Structured plasma source supplied with $\mathrm{H}_{2}$ and doped with $10 \% \mathrm{~N}_{2}$ in the front for controlled injection of electron bunches into the laser-driven plasma wave.

variations of the drive laser. Such a surrogate model opens up unique possibilities to evaluate the experiment and allows us to explore strategies for future improvement.

The experiment was performed at the LuX plasma accelerator [25,26] at DESY [Fig. 1(a)]. The Ti:sapphire laser system ANGUS delivered pulses at $1 \mathrm{~Hz}$, with an ontarget energy of $2.6 \mathrm{~J}(0.6 \% \mathrm{rms}$ stability) and a pulse duration of 34 fs FWHM ( 2\% rms stability), corresponding to a peak power of $69 \mathrm{TW}$. The laser was focused by a $2 \mathrm{~m}$ focal length off-axis parabolic (OAP) mirror at $f / 25$ to a $25 \mu \mathrm{m}$ FWHM spot size (0.85 Strehl ratio) inside a gasfilled target, resulting in a peak normalized vector potential of $a_{0}=2.1$. Online laser diagnostics measured the pulse energy and spectrum before the compressor as well as the near- and far-field and the wavefront behind the final focusing mirror.

Electron bunches leaving the plasma were either focused by a compact electromagnetic quadrupole doublet or allowed to naturally diverge in a free drift to a permanent magnet dipole spectrometer. The spectrometer had an energy resolution $\leq 1 \%$ between 220 and $310 \mathrm{MeV}$ for unfocused beams with $\mathrm{rms}$ divergences $\leq 2 \mathrm{mrad}$ and $\sim 0.1 \%$ for energies focused onto the screen. Transverse beam profiles were measured on a retractable scintillator screen and the center of mass and bunch charge were noninvasively measured by a cavity beam position monitor (BPM). The latter was used to calibrate the spectrometer [27].

The plasma source [Fig. 1(b)] consisted of a micromachined structure of square-shaped channels $(500 \mu \mathrm{m}$ edge length), milled into a sapphire crystal. A first inlet continuously supplied the entrance of a 5-mm-long on-axis channel with a 90:10 mixture of hydrogen $\left(\mathrm{H}_{2}\right)$ and nitrogen $\left(\mathrm{N}_{2}\right)$. In this section, a high-quality electron bunch was produced from ionization injection and then accelerated to higher energies in a following section, which was supplied with pure hydrogen by a pair of inlets with equal mass flow. An integrated vacuum outlet separated both sections. Operation at a balanced pressure ratio prevented the diffusion of nitrogen, which resulted in the formation a short $(\sim 700 \mu \mathrm{m})$ transition region. Additional on-axis ports allowed a direct measurement of the static pressure at the mixed gas inlet $(19.9 \mathrm{mbar})$ and in the center of the hydrogen region (24.9 mbar). Based on the measured mass flow and pressure values, the gas distribution was derived from computational fluid dynamics simulations with ANSYS FLUENT, assuming turbulent flow and using the Chapman-Enskog kinetic theory to model the mixing of gas species.

The laser-plasma interaction was modeled with particlein-cell simulations using the spectral, quasicylindrical code FBPIC $[34,35]$. The measured radial laser intensity evolution was approximated by a flattened Gaussian beam [36]. To account for absolute measurement errors, we used an optimization algorithm that varied the simulation input parameters within the error of the experimentally measured parameters and, thereby, converged the simulation results to the experimental data [27].

In the experiment, key laser and plasma source parameters provided control over the initially injected phase space and its subsequent acceleration. The overall gas density scaled the nominal accelerating gradient and mainly defined the final beam energy. The dopant concentration not only changed the injection rate, but also affected the trapping process by influencing the plasma profile. Operation at a high concentration maximized the injection rate at low laser intensities, which improves the quality of the initial phase space. The longitudinal focus position $z_{\text {foc }}$ and the laser energy $E_{\text {laser }}$ both gave control over the amount of injected charge, yet, balancing their contributions was required to minimize the final energy spread. Tuning those parameters, we optimized the accelerator to a working point with optimal beam loading conditions.

Before presenting the measured data at this working point, we explain the injection and acceleration dynamics that result in optimal beam loading, based on the corresponding simulation shown in Fig. 2.

As the laser pulse is focused through the target $\left(z_{\mathrm{foc}}=4.75 \mathrm{~mm}\right)$, its leading edge preionizes the weakly bound energy levels of the gas atoms $\left(\mathrm{H}^{+}, \mathrm{N}^{1-5+}\right)$, forming a background plasma of density $n_{e} \simeq 9.6 \times 10^{17} \mathrm{~cm}^{-3}$. The inner electrons of nitrogen $\left(\mathrm{N}^{6,7+}\right)$, however, are tunnelionized only in regions of high laser intensity at later wake phases and, therefore, can get trapped [37]. Along the first density upramp, the evolution of the wakefield initially suppresses trapping of those electrons [Fig. 2(a)]. As the pulse reaches higher intensities, the plasma wave transitions to the blowout regime [38], and localized injection of electrons occurs over a short distance of $\sim 700 \mu \mathrm{m}$ along the density downramp, which lowers the trapping threshold by decreasing the wake phase velocity $[39,40]$. In combination with the decrease in nitrogen doping, this leads to the injection of a bunch with a ramped current profile that decreases from head to tail. Eventually, the tail of this profile gets truncated in the second density upramp and an 


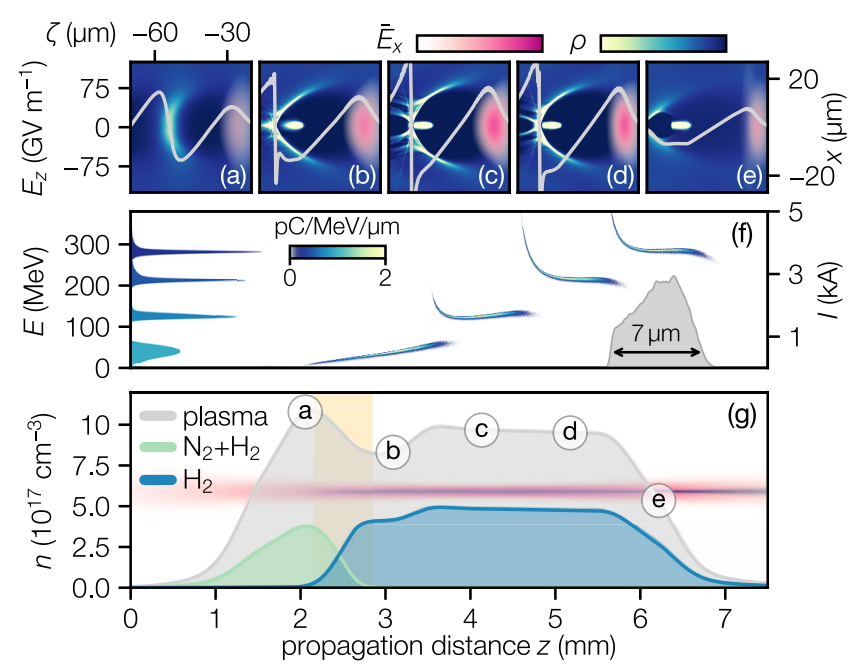

FIG. 2. Particle-in-cell simulation of the injection and acceleration process. (a)-(e) 2D snapshots $(\zeta=z-c t, x, y=0)$ of the laser, plasma wave, and electron bunch at different propagation distances $z$, showing the charge density $\rho$, laser field envelope $\bar{E}_{x}$, and on-axis longitudinal wakefield $E_{z}$. (f) Evolution of the longitudinal phase space $(\xi, E)$ corresponding to (b)-(e) and projections to the energy axis; Current profile at position (e). (g) Molecular gas $\left(10 \% \mathrm{~N}_{2}+90 \% \mathrm{H}_{2} ; 100 \% \mathrm{H}_{2}\right)$ and resulting plasma electron density profile; $z$ positions of (a)-(e) (markers); injection region (yellow), laser (red), and electron beam (blue) waist evolution.

isolated bunch with $49 \mathrm{pC}$ of charge distributed along a $\Delta \xi \simeq 7 \mu \mathrm{m}$ (23 fs) long quasitrapezoidal current profile (3 kA peak current) is subsequently accelerated to an energy of $288 \mathrm{MeV}$ throughout the 4-mm-long pure hydrogen plateau.

Immediately upon injection, the longitudinal phase space $(\xi, E)$ is positively correlated as a result of the retarded trapping of electrons, and beam loading along the current profile cancels the slope of the laser-driven wakefield [Fig. 2(b)]. Since the laser exceeds the critical power for relativistic self-focusing $\left(P / P_{c} \simeq 2\right)$ [2], a maximum intensity $a_{0} \simeq 3$ is reached in the center of the density plateau where the stronger blowout reverses the initial positive energy chirp [Fig. 2(c)]. Then, diffraction of the laser again leads to a decrease of the accelerating field until the beaminduced wakefield dominates at the end of the plasma [Figs. 2(d) and 2(e)]. At the exit of the target, the averaged dynamics result in a flattened core of the longitudinal phase space, with a projected relative energy spread of $\sim 1 \%$.

In the experiment, shot-to-shot laser fluctuations caused the injection and acceleration process to deviate from those optimal beam loading conditions, primarily via the amount of injected charge. However, by analyzing these variations, we can infer the underlying phase space dynamics directly from the measured data.

For this analysis, we recorded a series of 5207 consecutive shots. Events where no beam was generated or a laser or electron diagnostic was missing were excluded $(<3 \%$ of all shots). The electron bunches were unfocused and had a rms beam divergence of $(0.8 \pm 0.1)$ and $(0.6 \pm 0.2) \mathrm{mrad}$ in $x$ and $y$, respectively. The pointing jitter was 0.5 and $0.3 \mathrm{mrad}$ rms.

Figure 3 shows the measured (a) energy spectra and (b) bunch charges sorted by the median bunch energy, as well as the (c) characteristic spectrum in regions of $(A)$ strong, $(B)$ optimal, and $(C)$ weak beam loading.

At higher charges $(A)$, overly strong beam loading locally reversed the slope of the wakefield and imprinted a positive chirp onto the longitudinal phase space $(\xi, E)$. With decreasing charge, the decelerating beam loading effects got weaker, and effectively sheared the tail of the
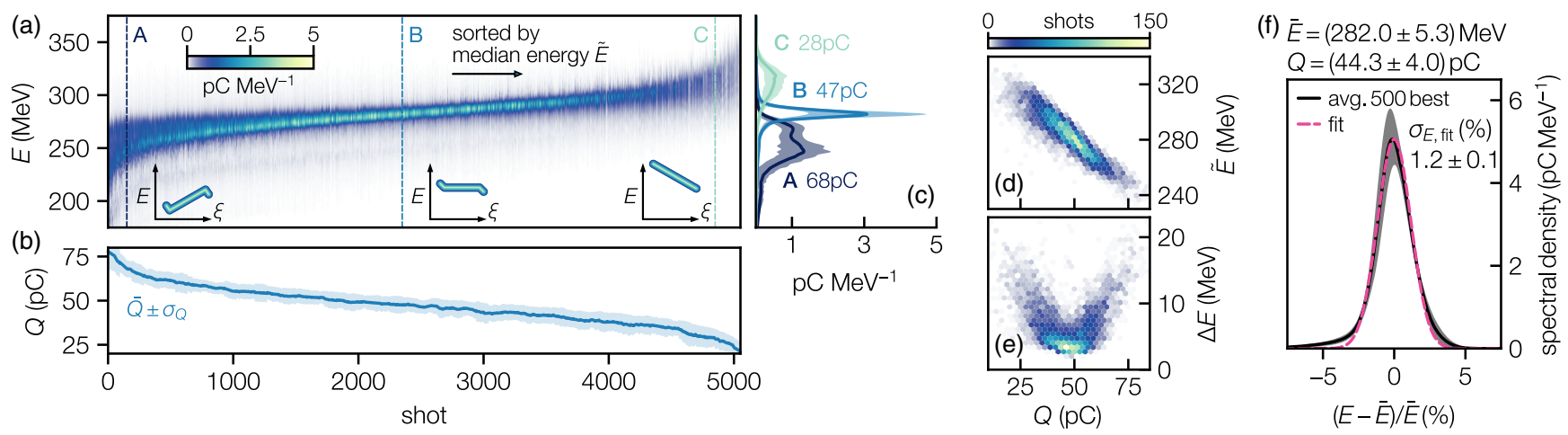

FIG. 3. Experimental results showing the phase space dynamics and optimal beam loading. (a) Measured series of energy spectra sorted by median energy $\tilde{E}$; (b) 100-shot moving average $\bar{Q}$ (solid line) and standard deviation $\pm \sigma_{Q}$ (shaded area) of the corresponding sorted charges $Q$. (c) 100-shot average spectrum (solid lines) and standard deviation (shaded area) in regions of strong $(A)$, optimal (B) and weak $(C)$ beam loading. Locations of $(A)-(C)$ and a schematic interpretation of the corresponding longitudinal phase spaces $(\xi, E)$ are shown in (a). Correlations of bunch charge $Q$ with (d) median energy $\tilde{E}$ and (e) energy spread (median absolute deviation) $\Delta E$. (f) Relative energy spectrum at optimal beam loading. 500-shot average (black solid line) and standard deviation (shaded area) of beams with lowest energy spread; Gaussian fit (magenta dashed line). Corresponding statistics of mean energy $\bar{E}$, rms energy spread $\sigma_{E, \text { fit }}$ and charge $Q$. 
bunch toward higher energies. The highest spectral density and lowest energy spread was reached when the core temporal slices overlapped in energy at the optimal load $(B)$. For lower charges $(C)$, effects from beam loading diminished causing the energy to monotonically increase from head to tail, as the electrons then mainly experienced the undisturbed laser-driven wakefield.

The same physics is reflected in the direct correlations of energy and energy spread with charge, Figs. 3(d) and 3(e). An increase in charge $Q$ linearly decreased the median energy, $\tilde{E}=(342-1.25 \times Q[\mathrm{pC}]) \mathrm{MeV}$ and resulted in a distinct $\mathrm{V}$-shaped distribution of the energy spread $\Delta E$ around a minimum at $\sim 47 \mathrm{pC}$. Note that we use the median absolute deviation as a robust statistical measure of the energy spread to account for the differently shaped spectra.

To fully characterize the accelerator performance at the optimal load, the measurement was repeated with the bunches focused onto the spectrometer screen. Figure 3(f) presents the average beam quality of the 500 shots with lowest energy spread, i.e., about $10 \%$ of this dataset (5130 consecutive shots), which we consider representative for operation at optimal beam loading conditions. On average, these bunches had an energy of (282 \pm 5.3) $\mathrm{MeV}$ with $(44.3 \pm 4) \mathrm{pC}$ of charge. The relative energy spread was $(1.2 \pm 0.1) \% \mathrm{rms}$ (Gaussian fit) or $(7.1 \pm 0.8) \mathrm{MeV}$ FWHM. The peak spectral density was $(5.3 \pm 0.7) \mathrm{pC} \mathrm{MeV}^{-1}$.

An energy-resolved quadrupole scan [8] was performed to measure the transverse phase-space properties in the nondispersive (laser polarization) plane. Consistent with the injection mechanism and in agreement with simulations, the normalized emittance was $(1.9 \pm 0.3) \mathrm{mm} \mathrm{mrad}$ with a reconstructed rms source size and beam divergence of $(4.0 \pm 0.1) \mu \mathrm{m}$ and $(0.9 \pm 0.1) \mathrm{mrad}$, respectively.

The beam loading efficiency at the optimal load was estimated from the simulation. We derived a wake-to-beam energy-transfer efficiency of $\sim 19 \%$ by relating the energy loss of the laser $(48 \mathrm{~mJ})$ to the energy gain of the electrons (9 $\mathrm{mJ})$ along the 2-mm-long plateau region. Higher efficiencies would require injecting more charge, by using longer bunches or by operating in a regime of stronger blowout.

For the remainder of this analysis, we again use the dataset of unfocused electron beams to study the origin of shot-to-shot instabilities. The measured correlations between laser and electron parameters [Figs. 4(a)-4(c)] reveal that the observed variations in charge, which lead to the beam loading variations, are directly related to fluctuations of the laser intensity at the point of injection. Predominantly, small variations of the wavefront defocus of only $6.9 \mathrm{~nm}$ rms translate to a longitudinal focus position jitter $\sigma_{z_{\text {foc }}}=162 \mu \mathrm{m} \mathrm{rms,} \mathrm{which} \mathrm{scales} \mathrm{the} \mathrm{intensity} \mathrm{and,}$ thus, the ionization and trapping rate along the density downramp [Figs. 2(a) and 2(b)]. This mechanism was verified by a scan of the longitudinal target position and

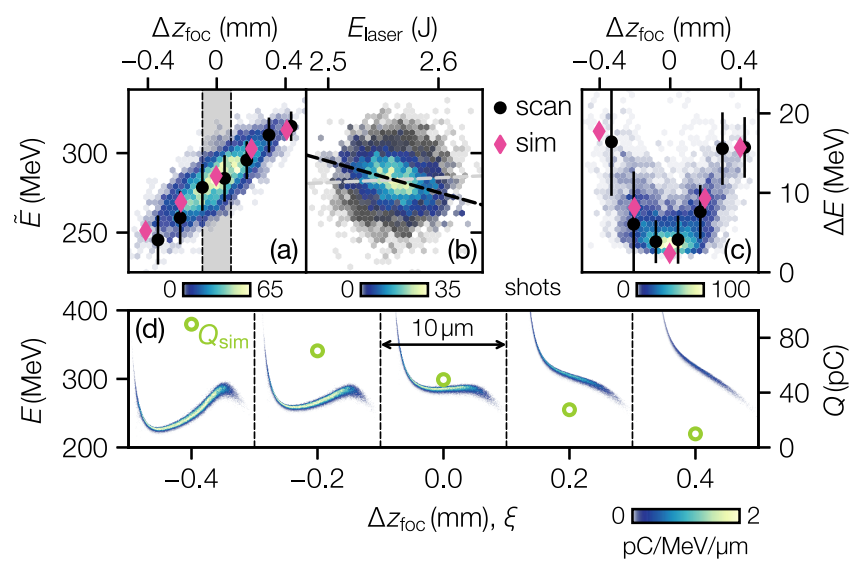

FIG. 4. Influence of the drive laser. Correlation of median electron energy $\tilde{E}$ with (a) laser focus position $\Delta z_{\text {foc }}$ and (b) pulse energy $E_{\text {laser }}$ with (colored; black dashed line) and without (grayed out) filtering the data by $\pm 0.5 \sigma_{z_{\text {foc }}}$ [gray area in (a)]. (c) Correlation of energy spread $\Delta E$ with $\Delta z_{\text {foc }}$. Overlaid are results from a longitudinal target position scan (black dots; 100-shot average) and particle-in-cell simulations (magenta diamonds). (d) Corresponding longitudinal phase spaces $(\xi, E)$ and charges $Q_{\text {sim }}$ (green circles) from the simulations.

reproduced in the simulations. Figure 4(d) shows how a downstream (upstream) shift of the focus position decreases (increases) the injected charge and, thereby, shears the final longitudinal phase space to higher (lower) energies.

Of course, other laser properties also vary the sensitive injection and acceleration process. However, because they have less influence or are more stable, their effect can remain hidden. For example, a subtle correlation between laser pulse energy $E_{\text {laser }}$ and electron energy $\tilde{E}$ [Fig. 4(b)] becomes visible only after filtering the data for shots within $\bar{z}_{\text {foc }} \pm 0.5 \sigma_{z_{\text {foc }}}$. An otherwise expected weak positive correlation of electron energy with laser energy due to an increase in wakefield strength is overcompensated by stronger beam loading due to an increase of injected charge.

Disentangling the interplay of hidden and nonlinear dependencies is a nontrivial task that motivates the application of machine learning [41] to build a multivariate regression model of the experiment. We trained an artificial neural network to predict, for each single shot, the beam energy, charge, and energy spread from 15 different laser parameters: the laser pulse energy, central wavelength and spectral bandwidth, position and pointing into the target, longitudinal focus position, and higher order wavefront aberrations. The network [27] consisted of an input (15 neurons), output (3 neurons, linear activation) and two hidden layers (30 neurons, rectifier activation) with a $20 \%$ dropout regularization. The mean absolute error was used as loss function. The first $80 \%$ of the chronologically ordered dataset were used to learn the hidden dependencies, while the following $20 \%$ served as a test dataset to evaluate the performance of the neural network. 


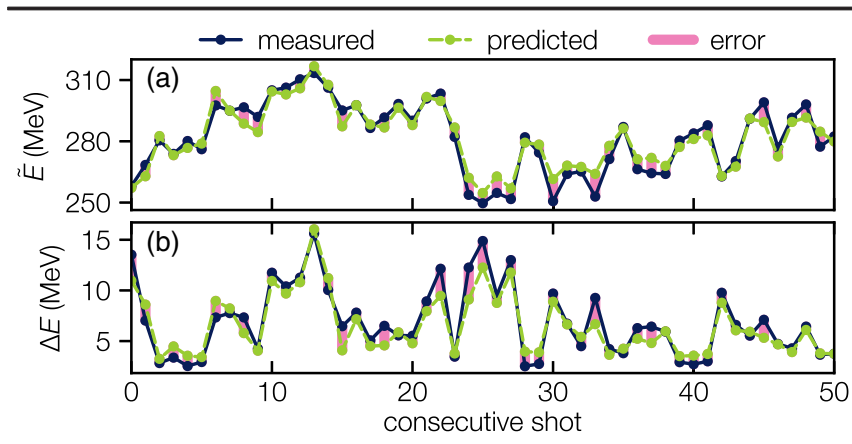

FIG. 5. Single-shot electron beam quality prediction by a neural network and from a set of input laser parameters. Measured (blue) and predicted (green) (a) median energy $\tilde{E}$ and (b) energy spread $\Delta E$ for a series of 50 consecutive shots. Prediction error (red).

Figure 5 demonstrates the predictive power of the model by comparing the measured and predicted energy $\tilde{E}$ and energy spread $\Delta E$. For better visualization of the singleshot predictive capabilities, we show only 50 out of the 1000 available consecutive shots [27]. Remarkably, the limited set of laser diagnostics is already sufficient to accurately determine the electron beam quality. Over the entire test dataset, the model achieves a coefficient of determination $\left(R^{2}\right)$ of $0.84,0.74$, and 0.73 for the energy, energy spread, and charge, respectively. These results indicate that we not only identified the main laser parameters affecting stability, but also that the plasma source itself seems not to be a dominant source of instability.

We can use the model to explore strategies for future improvement. For example, we can benchmark the effect of a hypothetical active feedback system operating at $1 \mathrm{~Hz}$ : One could envision stabilizing each laser parameter to a target value, by correcting for $75 \%$ of the deviation from the previous shot. In this case, the model predicts a reduction of the rms jitter of all electron beam properties by more than $28 \%$. This highlights the potential of fast feedback systems, especially in light of the upcoming transition to kilohertz repetition rates.

Similarly, we can quantify how specific improvements to the laser system could benefit the accelerator stability and derive guidelines for future laser development. For example, it seems feasible to (i) reduce the longitudinal focus position jitter by a factor of 4 to $41 \mu \mathrm{m}$ rms; (ii) halve the jitter of higher order wavefront aberrations to $\sim 2 \mathrm{~nm} \mathrm{rms}$; and (iii) halve the central wavelength jitter to $0.15 \mathrm{~nm}$ rms. With those improvements, the electron energy jitter would decrease by a factor of $\sim 3$ (then $1.9 \% \mathrm{rms}$ ) and $90 \%$ of all shots would have a relative energy spread $\Delta E / \tilde{E}<1.7 \%$.

The model can also be used to further optimize the beam quality. Within the fluctuations of the measurement, it already predicts a local minimum of the energy spread ( $\sim 10 \%$ smaller) at a slightly shifted working point. Wide-range online training of the model in future experiments could be used to efficiently find global optima.
In conclusion, we have demonstrated laser-plasma acceleration at optimal beam loading conditions, which resulted in a flattening of the average accelerating fields to $\sim 1 \%$ over a substantial fraction of the plasma wavelength $\left(\sim \lambda_{p} / 5\right)$. This enabled the production of $1.2 \%$ rms energy spread electron bunches at $282 \mathrm{MeV}$ with a peak spectral density of $5.3 \mathrm{pC} \mathrm{MeV}^{-1}$ (44 $\mathrm{pC}$ charge) and a transverse emittance of $1.9 \mathrm{~mm}$ mrad. Simulations indicate a beam loading efficiency of $\sim 19 \%$.

Applications demand highly reproducible beams, which motivated us to study the origin of shot-to-shot variations of the injection and acceleration process. Using machine learning, we could build a surrogate model of the experiment that accurately (average $R^{2}=0.77$ ) predicts how deviations from the optimal beam loading conditions dilute the single-shot beam quality as a function of key drive laser properties. The model identifies and quantifies sources of instability, serves as a basis for active feedback and global optimization techniques, or can simply be used as a noninvasive virtual diagnostic for the electron beam. We expect such models to become an essential tool for the realization of plasma accelerators with application-relevant stability and quality.

Further optimizing and scaling our experiment to the GeV-level would enable the per-mille energy spreads and tens-of-percent conversion efficiencies required by future applications. To reach this milestone, however, it is critical to increase the shot-to-shot stability, also because it ultimately limits the achievable level of control over the accelerating fields.

We appreciate the support of workshops and technical groups of University of Hamburg and DESY. We thank W. P. Leemans, C. A. Lindstrøm, R. Lehe, and C. Benedetti for discussions. This work was supported by BMBF Grants No. 05K19GUA, No. 05K19GUD, and computing time granted on the supercomputer JUWELS (Project No. CHHH20) at Jülich Supercomputing Centre.

Note added in proof.-After submitting this Letter, we used the developed plasma source and strategy for achieving optimal beam loading in a subsequent study to demonstrate autonomous tuning of the accelerator using Bayesian optimization. This work has since been published in [42].

*manuel.kirchen@desy.de

[1] T. Tajima and J. M. Dawson, Laser Electron Accelerator, Phys. Rev. Lett. 43, 267 (1979).

[2] E. Esarey, C. B. Schroeder, and W. P. Leemans, Physics of laser-driven plasma-based electron accelerators, Rev. Mod. Phys. 81, 1229 (2009).

[3] W. P. Leemans, B. Nagler, A. J. Gonsalves, C. Tóth, K. Nakamura, C. G. Geddes, E. Esarey, C. B. Schroeder, and S. M. Hooker, GeV electron beams from a centimetre-scale accelerator, Nat. Phys. 2, 696 (2006). 
[4] A. J. Gonsalves et al., Petawatt Laser Guiding and Electron Beam Acceleration to $8 \mathrm{GeV}$ in a Laser-Heated Capillary Discharge Waveguide, Phys. Rev. Lett. 122, 084801 (2019).

[5] O. Lundh, J. Lim, C. Rechatin, L. Ammoura, A. Ben-Ismaïl, X. Davoine, G. Gallot, J. P. Goddet, E. Lefebvre, V. Malka, and J. Faure, Few femtosecond, few kiloampere electron bunch produced by a laser-plasma accelerator, Nat. Phys. 7, 219 (2011).

[6] A. Buck, M. Nicolai, K. Schmid, C. M. Sears, A. Sävert, J. M. Mikhailova, F. Krausz, M. C. Kaluza, and L. Veisz, Real-time observation of laser-driven electron acceleration, Nat. Phys. 7, 543 (2011).

[7] G. R. Plateau, C. G. R. Geddes, D. B. Thorn, M. Chen, C. Benedetti, E. Esarey, A. J. Gonsalves, N. H. Matlis, K. Nakamura, C. B. Schroeder, S. Shiraishi, T. Sokollik, J. van Tilborg, C. Toth, S. Trotsenko, T. S. Kim, M. Battaglia, T. Stöhlker, and W. P. Leemans, Low-Emittance Electron Bunches from a Laser-Plasma Accelerator Measured using Single-Shot X-Ray Spectroscopy, Phys. Rev. Lett. 109, 064802 (2012).

[8] R. Weingartner, S. Raith, A. Popp, S. Chou, J. Wenz, K. Khrennikov, M. Heigoldt, A. R. Maier, N. Kajumba, M. Fuchs, B. Zeitler, F. Krausz, S. Karsch, and F. Grüner, Ultralow emittance electron beams from a laser-wakefield accelerator, Phys. Rev. ST Accel. Beams 15, 111302 (2012).

[9] A. R. Maier, A. Meseck, S. Reiche, C. B. Schroeder, T. Seggebrock, and F. Grüner, Demonstration Scheme for a Laser-Plasma-Driven Free-Electron Laser, Phys. Rev. X 2, 031019 (2012).

[10] S. Corde, K. Ta Phuoc, G. Lambert, R. Fitour, V. Malka, A. Rousse, A. Beck, and E. Lefebvre, Femtosecond x rays from laser-plasma accelerators, Rev. Mod. Phys. 85, 1 (2013).

[11] C. B. Schroeder, E. Esarey, C. G. R. Geddes, C. Benedetti, and W. P. Leemans, Physics considerations for laser-plasma linear colliders, Phys. Rev. ST Accel. Beams 13, 101301 (2010).

[12] C. B. Schroeder, E. Esarey, C. Benedetti, and W. P. Leemans, Efficiency considerations for high-energy physics applications of laser-plasma accelerators, AIP Conf. Proc. 1777, 020001 (2016).

[13] S. Van der Meer, Improving the power efficiency of the plasma wakefield accelerator, CERN Tech. Report No. PS-85-65, 1985.

[14] T. C. Katsouleas, S. Wilks, P. Chen, J. M. Dawson, and J. J. $\mathrm{Su}$, Beam loading in plasma accelerators, Part. Accel. 22, 81 (1987).

[15] M. Tzoufras, W. Lu, F. S. Tsung, C. Huang, W. B. Mori, T. Katsouleas, J. Vieira, R. A. Fonseca, and L. O. Silva, Beam Loading in the Nonlinear Regime of Plasma-Based Acceleration, Phys. Rev. Lett. 101, 145002 (2008).

[16] M. Tzoufras, W. Lu, F. S. Tsung, C. Huang, W. B. Mori, T. Katsouleas, J. Vieira, R. A. Fonseca, and L. O. Silva, Beam loading by electrons in nonlinear plasma wakes, Phys. Plasmas 16, 056705 (2009).

[17] C. Rechatin, X. Davoine, A. Lifschitz, A. B. Ismail, J. Lim, E. Lefebvre, J. Faure, and V. Malka, Observation of Beam Loading in a Laser-Plasma Accelerator, Phys. Rev. Lett. 103, 194804 (2009).

[18] C. Rechatin, J. Faure, X. Davoine, O. Lundh, J. Lim, A. Ben-Ismal, F. Burgy, A. Tafzi, A. Lifschitz, E. Lefebvre, and V. Malka, Characterization of the beam loading effects in a laser plasma accelerator, New J. Phys. 12, 045023 (2010).

[19] E. Guillaume, A. Döpp, C. Thaury, A. Lifschitz, J.-P. Goddet, A. Tafzi, F. Sylla, G. Iaquanello, T. Lefrou, P. Rousseau, K.T. Phuoc, and V. Malka, Physics of fully-loaded laser-plasma accelerators, Phys. Rev. ST Accel. Beams 18, 061301 (2015).

[20] J. P. Couperus, R. Pausch, A. Köhler, O. Zarini, J. M. Krämer, M. Garten, A. Huebl, R. Gebhardt, U. Helbig, S. Bock, K. Zeil, A. Debus, M. Bussmann, U. Schramm, and A. Irman, Demonstration of a beam loaded nanocoulombclass laser wakefield accelerator, Nat. Commun. 8, 487 (2017).

[21] J. Götzfried, A. Döpp, M. F. Gilljohann, F. M. Foerster, H. Ding, S. Schindler, G. Schilling, A. Buck, L. Veisz, and S. Karsch, Physics of High-Charge Electron Beams in LaserPlasma Wakefields, Phys. Rev. X 10, 041015 (2020).

[22] A. Pak, K. A. Marsh, S. F. Martins, W. Lu, W. B. Mori, and C. Joshi, Injection and Trapping of Tunnel-Ionized Electrons into Laser-Produced Wakes, Phys. Rev. Lett. 104, 025003 (2010).

[23] C. McGuffey, A. G. R. Thomas, W. Schumaker, T. Matsuoka, V. Chvykov, F. J. Dollar, G. Kalintchenko, V. Yanovsky, A. Maksimchuk, K. Krushelnick, V. Y. Bychenkov, I. V. Glazyrin, and A. V. Karpeev, Ionization Induced Trapping in a Laser Wakefield Accelerator, Phys. Rev. Lett. 104, 025004 (2010).

[24] B. B. Pollock et al., Demonstration of a Narrow Energy Spread, $0.5 \mathrm{GeV}$ Electron Beam from a Two-Stage Laser Wakefield Accelerator, Phys. Rev. Lett. 107, 045001 (2011).

[25] N. Delbos, C. Werle, I. Dornmair, T. Eichner, L. Hübner, S. Jalas, S. W. Jolly, M. Kirchen, V. Leroux, P. Messner, M. Schnepp, M. Trunk, P. A. Walker, P. Winkler, and A. R. Maier, LUX-A laser-plasma driven undulator beam line, Nucl. Instrum. Methods Phys. Res., Sect. A 909, 318 (2018).

[26] A. R. Maier, N. M. Delbos, T. Eichner, L. Hübner, S. Jalas, L. Jeppe, S. W. Jolly, M. Kirchen, V. Leroux, P. Messner, M. Schnepp, M. Trunk, P. A. Walker, C. Werle, and P. Winkler, Decoding Sources of Energy Variability in a Laser-Plasma Accelerator, Phys. Rev. X 10, 031039 (2020).

[27] See Supplemental Material at http://link.aps.org/ supplemental/10.1103/PhysRevLett.126.174801 for technical details of the neural network, the experimental setup, and the particle-in-cell simulations, which includes Refs. [28-33].

[28] F. Chollet et al., Keras (2015).

[29] F. Pedregosa, G. Varoquaux, A. Gramfort, V. Michel, B. Thirion, O. Grisel, M. Blondel, P. Prettenhofer, R. Weiss, V. Dubourg, J. Vanderplas, A. Passos, D. Cournapeau, M. Brucher, M. Perrot, and Édouard Duchesnay, Scikit-learn: Machine Learning in PYTHON, J. Mach. Learn. Res. 12, 2825 (2011).

[30] D. Lipka et al., Very first experience with the standard diagnostics at the European XFEL, in Proceedings of 8th International Particle Accelerator Conference (JACoW, Geneva, 2017), p. 180.

[31] P. Winkler, Emittance measurements at laser-wakefield accelerators, Ph.D. thesis, Universität Hamburg, 2019. 
[32] M. Chen, E. Cormier-Michel, C. Geddes, D. Bruhwiler, L. Yu, E. Esarey, C. Schroeder, and W. Leemans, Numerical modeling of laser tunneling ionization in explicit particle-incell codes, J. Comput. Phys. 236, 220 (2013).

[33] D. R. Jones, M. Schonlau, and W. J. Welch, Efficient global optimization of expensive black-box functions, J. Global Optim. 13, 455 (1998).

[34] R. Lehe, M. Kirchen, I. A. Andriyash, B. B. Godfrey, and J.L. Vay, A spectral, quasi-cylindrical and dispersion-free Particle-In-Cell algorithm, Comput. Phys. Commun. 203, 66 (2016).

[35] M. Kirchen, R. Lehe, S. Jalas, O. Shapoval, J.-L. Vay, and A. R. Maier, Scalable spectral solver in Galilean coordinates for eliminating the numerical Cherenkov instability in particle-in-cell simulations of streaming plasmas, Phys. Rev. E 102, 013202 (2020).

[36] M. Santarsiero, D. Aiello, R. Borghi, and S. Vicalvi, Focusing of axially symmetric flattened Gaussian beams, J. Mod. Opt. 44, 633 (1997).

[37] M. Chen, E. Esarey, C. B. Schroeder, C. G. R. Geddes, and W. P. Leemans, Theory of ionization-induced trapping in laser-plasma accelerators, Phys. Plasmas 19, 033101 (2012).

[38] W. Lu, C. Huang, M. Zhou, W. B. Mori, and T. Katsouleas, Nonlinear Theory for Relativistic Plasma Wakefields in the Blowout Regime, Phys. Rev. Lett. 96, 165002 (2006).

[39] S. Bulanov, N. Naumova, F. Pegoraro, and J. Sakai, Particle injection into the wave acceleration phase due to nonlinear wake wave breaking, Phys. Rev. E 58, R5257 (1998).

[40] A. J. Gonsalves, K. Nakamura, C. Lin, D. Panasenko, S. Shiraishi, T. Sokollik, C. Benedetti, C. B. Schroeder, C. G. R. Geddes, J. van Tilborg, J. Osterhoff, E. Esarey, C. Toth, and W. P. Leemans, Tunable laser plasma accelerator based on longitudinal density tailoring, Nat. Phys. 7, 862 (2011).

[41] G. Carleo, I. Cirac, K. Cranmer, L. Daudet, M. Schuld, N. Tishby, L. Vogt-Maranto, and L. Zdeborová, Machine learning and the physical sciences, Rev. Mod. Phys. 91, 045002 (2019).

[42] S. Jalas, M. Kirchen, P. Messner, P. Winkler, L. Hübner, J. Dirkwinkel, M. Schnepp, R. Lehe, and A. R. Maier, Bayesian Optimization of a Laser-Plasma Accelerator, Phys. Rev. Lett. 126, 104801 (2021). 